XLVII.

Chemische Notizen.

Mitgetheilt

von

Otto Iinné Iramann.

1) Ueber die Sulfamylschwefelsäure.

Die nachstehende Untersuchung über diese Säure wurde von Herrn E. G e r a the w o h I aus Ebersbach im hiesigen UniversitätsLaboratorium ausgeführt.

Die Sulfamylschwefelsäure ist das Hauptproduct der Zersetzung des Amylmercaptans durch Salpetersäure. Sie wurde auf ganz dieselbe Weise erhalten, wie Löwig und Weidmann (Pogg. Ann. Bd. XLVII u. XLIX), so wie H. Kopp (Ann. ḋ. Chem. u. Pharm. Bd. XXXV) die analoge Aethylverbindung, die Sulfäthylschwefelsäure, erhielten.

Das zur Darstellung verwandte Amylmercaptan wurde anfänglich aus rohem, nur vorher mit Wasser behandeltem Kartoffelfuselöle erhalten, durch Behandlung desselben mit Schwefelsäure, Sättigen mit kohlensaurem Kali, Zusetzen von kaustischem Kali, wobei sich ein braungefärbtes $0 \mathrm{el}$ ausschied, das auf der Flüssigkeit schwamm und abgenommen wurde, Einleiten von Schwefelwasserstoffgas in die Lösung und nachherige Destillation. Es wurde jedoch hierdurch nur ein ziemlich unreines Product gewonnen.

Bei einer zweiten Bereitung wurde amylschwefelsaures Kali aus reinem, bei $131^{\circ}$ siedendem Fuselöle dargestellt und zu der Auflösung dieses Salzes kaustisches Kali gesetzt, wobei sich eine Ausscheidung des braunen Oeles nicht zeigte. Mit Schwefelwasserstoffgas behandelt und destillirt, wurde so ein reineres Product erhalten.

Die Producte beider Darstellungen zeigten bei der Einwirkung der Salpetersäure dieselben Erscheinungen.

Behufs der vollständigen Reinigung des rohen Diercaptans wurde ein Versuch angestellt, dasselbe mit Quecksilberoxyd in Amylmercaptid zu verwandeln und aus diesem durch Chlorwasserstoffsäure das Amylmercaptan wieder abzuscheiden. Allein die Abscheidung des Mercaptans auf diese Weise wollte nicht gelin- 
gen, die Zersetzung war immer nur partiell und lieferte eine sehr unbedeutende Ausbeute.

Ein anderer Versuch, das Amylmercaptid durch Schwefelwasserstoffgas zu zersetzen, zeigte wenigstens die Möglichkeit, obgleich die gewonnene Menge von Mercaptan ebenfalls nicht bedeutend war.

Es wurde das Mercaptid in einer Glasröhre mit Glaspulver gemengt und Schwefelwasserstoffgas hindurchgeleitet, während man die Röhre vorsichtig gelinde erwärmte. Die Röhre selbst endete in eine Spitze, und das freigewordene Mercaptan destillirte in eine kleine Vorlage über, es enthielt noch sehr viel Schwefelwasserstoff und musste wiederholt für sich destillirt werden*).

Die Einwirkungen der Salpelersäure auf das Amylmercaptan und die dabei sich kundgebenden Erscheinungen sind im Allgemeinen dieselben, wie sie bei der Aethylverbindung beschrieben sind, und es liess sich erwarten, dass auch die entsprechenden Zersetzungsproducte daraus hervorgehen würden.

Beim Zusammenbringen des Amylmercaptans mit reiner Salpetersäure von 1,25 spec. Gew. zeigte sich anfänglich in der Kälte keine Reaction. Beim gelinden Erwärmen der Säure verändert die auf derselben schwimmende Mercaptanschicht ihre Farbe, sie wird roth, und bald darauf beginnt eine sehr heftige Oxydation unter starker Wärmeentwickelung und Bildung von salpetrigsauren Dämpfen. Nach längerem Stehen oder bei Anwendung von stärkerer Säure zeigen sich auch in der Kälte dieselben Erscheinungen. Gelinde Wärme beschleunigt nur die Einwirkung. Man darf das Mercaptan nur in sehr kleinen Quantitäten vorsichtig der Säure zusetzen, da bei zu heftiger Einwirkung ein grosser Theil des Mercaptans mit den Dämpfen fortgerissen wird. 'Die 0xydation wurde in einer kleinen tubulirten Retorte vorgenommen, um von den entweichenden Producten noch etwas in der Vorlage zurückhalten zu können. Hierbei ist grosse Vorsicht nöthig, um das Zersprengen der Retorte zu hindern.

Es wird so lange Mercaptan zugesetzt, als sich noch 0xydationserscheinungen beim Erwärmen zeigen.

*) Der Siedepunct des anf diese Weise erbaltenen Mercaptans lag bei 117-118 Grad, bei derselben Temperatur also, welche $\mathrm{Kratzscb,} \mathrm{s.} \mathrm{d.}$ Journ. XXXI. 1, angegeben hat, während B a lard später den Siedepunct zn 125 Grad angegeben hat. 
Nach beendigter Einwirkung hat man in der Retorte zwei Flüssigkeiten, eine saure Lösung und ein obenauf schwimmendes öliges Product von hellgelber Farbe.

Diese ölige Schicht wurde entfernt, und in der zurückbleibenden Flüssigkeit hat man nur ein Gemenge von Salpetersäure und Sulfamylschwefelsäure. Bei Anwendung einer Salpetersäure von 1,25 spec. Gew. ist die Bildung von Schwefelsäure nur gering.

Das Gemenge wird auf dem Wasserbade abgedampft, bis der Geruch nach Salpetersäure oder salpetriger Säure verschwunden ist. Es bleibt dann ein dickflüssiger, sirupartiger klarer farbloser Rückstand von unreiner Sulfamylschwefelsäure.

Sie kann in diesem $\mathbf{Z}$ ustande zur Darstellung der meisten Salze verwendet werden, da beim Auflösen der Salze in siedendem Alkohol, woraus sie beim Erkalten krystallisiren, die etwa beigemengten schwefelsauren Verbindungen ungelöst zurückbleiben.

Ganz reine Säure kann man erhalten, wenn man die beim $A b$ dampfen zurückbleibende Säure mit Wasser verdünnt, alsdann mit kohlensaurem Bleioxyd sättigt, das gebildete schwefelsaure Bleioxyd abfiltrirt und aus dem in der Auflösung befindlichen sulfamylschwefelsauren Bleioxyde mittelst Schwefelwasserstoff die Sulfamylschwefelsäure frei macht, welche nach dem Abdampfen auf dem Wasserbade wieder im dickflüssigen Zustande zurückbleibt und nun keine Schwefelsäure mehr enthält.

Die Sulfamylschwefelsäure besitzt einen stark sauren Geschmack und einen eigenthümlichen Geruch. An der Luft zieht sie Wasser an. Eine Bildung von Krystallen in derselben konnte selbst bei längerem Stehen über Schwefelsäure im Iuftleeren Raume nicht beobachtet werden. Die Salze dieser Säure können theils durch Auflösen der kohlensauren Basen in der freien Sïure, theils durch Zersetzung des Barytsalzes durch die schwefelsauren Salze der Basen dargestellt werden. Sie besitzen in ihren äusseren Eigenschaften die grösste Aehnlichkeit mit den Salzen der Sulfäthylschwefelsäure und sind wie diese leicht krystallisirt zu erhalten.

\section{Sulfamylschwefelsaurer Baryt.}

Dieses Salz wurde aus der rohen Säure dargestellt, durch Sättigen derselben mit kohlensaurem Baryt. Schwefelsaurer und überschüssiger kohlensaurer Baryt wurden ahfiltrirt, nnd aus der Lösung krystallisirte beim Abdampfen auf dem Wasserbade bis Journ. f. prakt. Chemie. Xxxiv, 8. 
zum Krystallisationspuncte das Barytsalz in farblosen, durchsichtigen, fettig anzufühlenden Blättchen, welche durch Umkrystallisiren in Alkohol gereinigt warden. Die Darstellungsweise zeigt schon, dass das Salz in Wasser und Weingeist löslich ist; die Lüslichkeit ist grösser in der Wärme als in der Kälte, daher das Salz aus der Lösung beim Erkalten herauskrystallisirt. $10 \mathrm{Th}$. Wasser lösen $1 \mathrm{Th}$. Salz bei $19^{\circ} \mathrm{C}$. Auf dem Wasser zeigte der sulfamylschwefelsaure Baryt dieselben Bewegungen wie der amilenschwefelsaure oder der buttersaure Baryt. Das Salz vertrïgt eine ziemlich hohe Temperatur; bei $160^{\circ}$ blieb dasselbe noch unverändert, es verliert jedoch schon bei $100^{\circ}$ Wasser.

Die Analyse dieses Salzes ergab Folgendes.

Die Substanz wurde stets bei $120^{\circ}$ im Luftbade mittelst der Handluftpumpe getrocknet.
I. 1624 Substanz gaben $863 \dddot{\mathrm{S}}$ B̈a oder 34,90 B்a
II. $955-\quad-508--34,93-$
III. $1276-\quad-679--34,95-$
IV. $1071-\quad-574--35,19-$
V. $1153-\quad-613--34,92-$
VI. $505--268-\frac{34,86-}{\text { Im Mittel } 34,96 .}$

Ferner gaben, bei $120^{\circ}$ getrocknet:

I. 1086 Substanz (blos mit Kupferoxyd verbrannt)

$$
\begin{aligned}
1047 \text { Kohlensäure } & =26,29 \frac{\mathrm{o}}{0} \mathrm{C}, \\
488 \text { Wasser } & =4,99 \mathrm{H} .
\end{aligned}
$$

II. 860 Subst. (mit Kupferoxyd im Sauerstoffstrome verbrannt) 860 Kohlensäure $=27,33 \mathrm{C}$, 390 Wasser $=5,00 \mathrm{H}$.

III. 521 Subst. (blos mit Kupferoxyd) 510 Kohlensäure $=26,70 \mathrm{C}$, 233 Wasser $=4,97 \mathrm{H}$.

IV. Eine vierte Analyse, mit Sauerstoff angestellt, von welcher die Detailangaben zufällig verloren gegangen sind, gab folgende Procente:

$$
\begin{aligned}
& =27,618 \mathrm{c}, \\
& =5,183 \mathrm{H} .
\end{aligned}
$$

Der Schwefel wurde durch Glühen eines Gemenges der Substanz mit kohlensaurem Baryt und Salpeter bestimmt. 
I. 864 sulfamylschwefelsaurer Baryt gaben :

$949 \dddot{\mathrm{S}} \mathrm{B} \mathrm{a}=15,077 \frac{0}{0}$ Schwefel.

II. 595 Substanz $669-=15,4 \quad-$

III. $670-762-=15,5$ - -

Aus diesen Resultaten lässt sich für das bei $120^{\circ}$ getrocknete Barytsalz eine Formel ableiten, welche der von $L$ öwig für die Aethylverbindung angegebenen ganz analog ist und sich von dieser nur durch 1 Atom Wasser unterscheidet. Sie ist nämlich:

$$
\begin{aligned}
& \mathrm{O}_{10} \mathrm{H}_{22} \mathrm{O}_{4} \mathrm{~S}_{2}+\mathrm{Ba} 0+\mathrm{H}_{2} 0 \text {. } \\
& \text { 氜 乎 }
\end{aligned}
$$

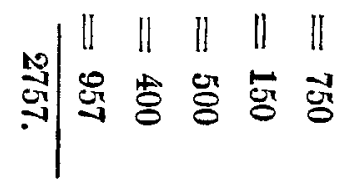

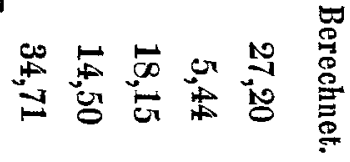

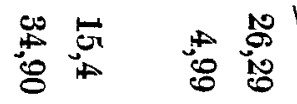

$$
\begin{aligned}
& \text { 苾 }
\end{aligned}
$$

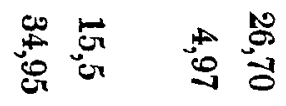

$$
\begin{aligned}
& \text { 点 } \\
& \text { vor } \\
& \text { 点 } \\
& \text { 幽 }
\end{aligned}
$$

Das berechnete Atomgewicht der Säure ist 1800, das aus den Analysen des Barytsalzes sich ergebende ist 1780. 


\section{Sulfamylschwefelsaures Bleioxyd.}

Das Salz wurde dargestellt durch Sättigen der Säure mit kohlensaurem Bleioxyd, Abdampfen bis zur Krystallisation, Auflösen in Weingeist und Umkrystallisiren. Das Salz krystallisirt in farblosen strahlig blättrigen Gruppen beim sehr langsamen Anschiessen aus nicht zu concentrirten Lösungen. Ist die Lösung sehr concentrirt, so erstarrt sie durch die ganze Masse, wie diess auch beim Baryt- und Silbersalze der Fall ist.

Bis $120^{\circ}$ erhitzt, verliert das Salz Wasser, erleidet aber sonst keine Veränderung; 1180 auf frischem Papier getrockneten Salzes verloren, bis zu $120^{\circ}$ erhitzt, 277 Wasser, was ungefähr 9 Atomen entspricht. Bei höherer Temperatur aber bräunt es sich und es entwickeln sich Dämpfe von höchst unangenehmem Geruche. Es brennt, wie das Barytsalz, mit bläulicher Schwefelflamme.

0,994 Substanz, bei $100-110^{\circ}$ im luftleeren Raume getrocknet, in Wasser gelöst und die Lösung mit oxalsaurem Ammoniak gefällt, gaben nach dem Verbrennen des oxalsauren Bleioxyds 0,434 Bleioxyd $=43,64 \frac{0}{0}$. Hieraus ergiebt sich das Atomgewicht zu 1799,35. - 1,289 Substanz, bei $100^{\circ}$ im luftleeren Raume getrocknet, gaben, auf dieselbe Weise bestimmt, 0,564 Bleioxyd = 43,75\% oder das Atomgewicht der Sulfamylschwefelsäure zu 1792.

Bei einer Verbrennung im Sauerstoffapparate gaben von der bei $100^{\circ}$ im Iuftleeren Raume getrockneten Substanz:

$$
\begin{aligned}
& 0,412 \mathrm{Grm} . \begin{array}{c}
363 \text { Kohlensäure }=24,02 \% \\
172 \text { Wasser }=4,64-
\end{array} \\
& \text { Berechnet. Gefunden. } \\
& \mathrm{C}_{10}=750=23,48 \quad 24,02 \\
& \mathrm{H}_{24}=150=4,69 \quad 4,64 \\
& \mathrm{~S}_{2}=400 \\
& \mathrm{Os}=500 . \\
& \mathrm{PbO}=\frac{1394,5=43,65}{3194,5}
\end{aligned}
$$

\section{Sulfamylschwefelsaures Silberoxyd.}

Ebenfalls durch Sättigen der Säure mit kohlensaurem Silberoxyd erhalten, krystallisirt beim gehörigen Concentrationsgrade in sehr schönen farblosen rhombischen Tafeln, in einer zu weit ein- 
gedampften Lösung aber erstarrle es zu einer amorphen Gallerte, ganz dem geronnenen Eiweiss ähnlich, welche unter dem Mikroskope bei 250facher Vergrösserung wie ein aus feinen verwebten Haaren bestehender Filz erschien. Dasselbe Salz wurde wieder aufgelöst in Wasser und krystallisirte alsdann aus einer weniger concentrirten Lösung.

Die Analyse des krystallisirten Silbersalzes gab keine befriedigenden Resultate, was wohl der nicht vollkommenen Reinheit desselben zugeschrieben werden muss.

$1,401 \mathrm{Grm}$. bei $100^{\circ}$ getrocknetes Salz gaben nach dem Verbrennen 0,615 Silber, oder 660,5 Silberoxyd $=47,13 \%$.

$1,217 \mathrm{Grm}$., ebenfalls bei $100^{\circ}$ getrocknet, gaben, als Chlorsilber bestimmt, 0,709 Chlorsilber $=0,574$ Silbero $x y d=47,13 \frac{\mathrm{o}}{0}$.

Aus beiden Bestimmungen leitet sich für das Atomgewicht der Säure zufällig dieselbe Zahl 1626 ab.

Die Formel der wasserfreien Säure würde 1687,5 fordern.

Zwei Verbrennungen im Sauerstoffapparate gaben folgende Resultate (die Substanz war bei $100^{\circ}$ im luftleeren Raume getrocknet) :

$$
\begin{aligned}
0,870 \mathrm{Grm} \text {. Substanz } 0,686 \text { Kohlensäure } & =21,49 \frac{\mathrm{O}}{\mathrm{C}} \\
0,317 \text { Wasser } & =4,02-\mathrm{H} . \\
0,930-\mathrm{0,718} \text { Kohlensäure } & =21,064 \frac{\mathrm{o}}{\mathrm{C}}, \\
0,331 \text { Wasser } & =3,960 \cdot \mathrm{H} .
\end{aligned}
$$

$$
\begin{array}{lccc} 
& \text { Berechnet. } & \multicolumn{2}{c}{\text { Gefunden. }} \\
\mathrm{C}_{10}=23,89 & 21,49 & 21,064 \\
\mathrm{H}_{22}=4,38 & 4,02 & 3,960 \\
\mathrm{~S}_{22}=12,745 & -- & - \\
\mathrm{O}_{4}=12,745 & - & - \\
\mathrm{Ag} .0=46,23 & \mathbf{4 7 , 1 3} & 47,13 .
\end{array}
$$

Sulfamylschwefelsaures Kali, sulfamylschwefelsaures Ammoniak und sulfamylschwefelsaurer Kalk sind in farblosen Bliittchen krystallisirbare, in Wasser und Weingeist lösliche Salze. Das Kupfersalz krystallisirt in blaugrünen Tafeln, welche schon über Schwefelsäure Wasser verlieren und undurchsichtig werden.

Die bei der Oxydation des Mercaptans mit Salpetersüure sich ausscheidende ölige Flüssigkeit schien durchaus keine constante Verbindung, sondern ein Gemenge zu sein von verschiedener Beschaffenheit, je nach den Umständen bei der 0xydation. Von einer 
Bereitung wurde ein Oel gewonnen, welches auf Wasser schwamm; eine andere Bereitung ergab ein Product, das schwerer war als Wasser. Beide aber waren in nicht hinreichenden Quantitäten vorhanden zu einer genauen Untersuchung. Indessen wurde eine Analyse der leichten Flüssigkeit ausgeführt, welche die Zusammensetzung gab:

$$
\begin{aligned}
& \mathbf{C}=56,02 \frac{\mathbf{0}}{\mathrm{o}} \\
& \mathrm{H}=10,38 \\
& \mathrm{~S}=9,24 \\
& \mathrm{D}=24,36 .
\end{aligned}
$$

2) Zusammensetzung des Chloritspathes (Chloritö̈d).

Im VI. Bande dieses Journals, S. 89, habe ich die Resultate einer von mir angestellten Untersuchung des von Fiedler beschriebenen Chloritspathes (Chloritoïd) mitgetheilt. Später hat v. Bonsdorff eine Analyse des Chloritoïds veröffentlicht (F. Rose's Reise nach dem Ural, I. 252, s. a. Rammels berg's Handwörterbuch der Mineralogie), welche ein ganz abweichendes Resultat lieferte, so dass Rammelsberg bemerkt, es scheine kaum glaublich, dass v. B onsdorff dasselbe Mineral untersucht haben solle, wogegen schon der von v. Bonsdorff gefundene bedeutende Wassergehalt spreche. Das von mir untersuchte Mineral war von dem Entdecker selbst mitgebracht und mit einer Etikette von seiner Hand versehen; ich kann also an der Identität desselben mit dem von Fiedler heschriebenen nicht zweifeln.

Ich stelle in Folgendem das Resultat meiner Analyse mit dem der v. Bons dorff'schen zusammen.

$$
\text { Erdmann. v. B. }
$$

$$
\text { 1. } 2 . \quad \text { Mittel. }
$$

Kieselerde $\quad 24,900 \quad 24,963 \quad 24,931 \quad 27,48$

Eisenoxydul 28,890 31,204 $30,047 \quad 27,05$

Thonerde $\quad 46,200 \quad 43,833 *) 45,016 \quad 35,57$

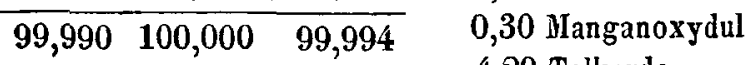

4,29 Talkerde

$\frac{6,95 \text { Wasser }}{101,64 .}$

*) Aas dejn Verluste bestimmt. 
Ich berechnete aus meiner Analyse nach dem Sanerstoffverhältnisse der Kieselerde, der Thonerde und des Eisenoxyduls, welches $=2: 3: 1$ ist, die Formeln:

$$
\begin{aligned}
\dot{\mathrm{F}} \mathrm{e}_{3} \dddot{\mathrm{S} i}+\dddot{\mathrm{AI}} 3 \dddot{\mathrm{S}} \mathrm{Si} \\
\text { oder } \dot{\mathrm{F}} \mathrm{e}_{3} \dddot{\mathrm{Al}}+2 \dddot{\mathrm{Al}} \dddot{\mathrm{S}} \mathrm{i} \text {. }
\end{aligned}
$$

v. Bons dorff berechnet aus seiner Analyse die Formel:

$$
\left.\begin{array}{l}
\dot{\mathrm{Fe}_{3}} \\
\dot{\mathrm{I}} \mathrm{g}_{3}
\end{array}\right\} \dddot{\mathrm{Si}}+\dddot{\mathrm{Ml}}_{2} \dddot{\mathrm{S} \mathrm{i}}+9 \dot{\mathrm{H}} .
$$

Auf meine Veranlassung hat Hr. Stud. chem. Ge rath ew ohl eine neue Analyse des von mir untersuchten Minerals im hiesigen Laboratorium angestellt.

Das Verhalten vor dem Löthrohre zeigte sich ganz so wie ich es beschrieben habe.

Im Glasrölrrchen erhitzt, giebt das Mineral unbedeutende Spuren von Wasser, welche jedoch nur vom anhängenden Eisenoxydhydrate herzurühren scheinen, da das später mit Säure behandelte Pulver sich ganz wasserfrei zeigt.

Offen geglüht, wird das Pulver dunkler und bei längerem Glühen endlich röthlich-braun, ohne zu schmelzen.

Die Kieselerde lässt sich vor dem Löthrohre sehr schwer erkennen, da das Mineral sich sowohl in kleinen Splittern als auch in Pulverform im Phosphorsalze bei anhaltendem Blasen endlich, obwohl langsam, doch vollstündig auflöst. Die Perle zeigt die Eisenreaction, aber kein Kieselskelett.

Mit Borax giebt es eine dunkelgrune Perle, die beim Erkalten heller wird.

Mit Soda auf Kohle schwillt das Mineral etwas an, auf Platinblech zeigen sich Spuren von Mangan.

Die qualitative Analyse auf nassem Wege zeigte, dass sich das Mineral durch Salz- oder Salpetersäure nicht aufschliessen lässt. Diese Säuren lösen das anhängende Eisenoxyd und die Thonerde auf, bis zuletzt ein Rückstand bleibt, welcher nicht weiter angegriffen wird.

Dieser Rückstand enthält nur Kieselsäure, Eisenoxydul mit Spuren von Mangan und Thonerde, namentlich aber keine Talkerde und kein Wasser.

Das zur quantitativen Analyse verwandte, feingeschlämmte Pulver wurde erst mit verdiinnter Salzsäure so lange behandelt 
bis sich nichts mehr darin löste, und dann bei $100 \mathrm{Grad}$ getrocknet.

Das Aufschliessen geschah mit einem Gemenge von kohlensaurem Kali und Natron, Auflösen mit Salzsäure u. s. w., wobei sich ganz die von mir beschriebenen Erscheinungen zeigten.

1,926 Grm. gaben :

$$
\begin{aligned}
0,470 \text { Kieselerde } & =24,40 \text { Proc. } \\
0,650 \text { Eisenoxyd } & =30,29-\text { oxydul } \\
0,877 \text { Thonerde } & =45,17-
\end{aligned}
$$

Diess stimmt so genau als möglich mit der früher von mir angestellten Analyse überein, wie folgende Zusammenstellung zeigt:

Gerathewohl. Erdmann (Mittel).

\begin{tabular}{llr} 
Kieselerde & 24,40 & 24,93 \\
Eisenoxydul & 30,29 & 30,05 \\
Thonerde & 45,17 & 45,02 \\
\cline { 2 - 3 } & 99,86 & $100,00$.
\end{tabular}

3) Analysent einiger Bronzen zu Maschinentheilen.

Die folgenden Analysen sind von meincm Assistenten, Herrn Emil $S$ chmid $t$ aus Dresden (gegenwärtig Lehrer der Chemie an der Gewerbschule zu Chemnitz), ausgeführt worden. Die analysirten Bronzen. waren sämmtlich solche, die sich als ganz vorzüglich dem Zwecke, zu welchem sie benutzt worden, entsprechend bewährt haben.

Der Gang der Analysen war der gewöhnliche: Auflösung in Salpetersäure, Eindampfen zur Trockne und Wiederauflösung in Wasser zum Behufe der Abscheidung des Zinnoxyds, Fällung mit Schwefelwasserstoff, Oxydation des Niederschlages durch Salpetersäure u.s.w. und Fällung des Kupferoxyds durch Kali; Abscheidung des Zinkes und Eisenoxyds durch SchwefelwasserstoffAmmoniak a. s. w. Das Blei wurde nach Abscheidung des Zinnoxyds durch Schwefelsäure, Eindampfen zur Trockne u. s. w. als schwefelsaures Bleioxyd bestimmt.

1) Metall zu den Achsenlagern einer englischen Locomotive, dessen ausgezeichnete Dauerhaftigkeit durch mehrjährige Erfahrung erprobt worden war. 


\begin{tabular}{lr} 
Zinn & $\mathbf{9 , 4 5}$ \\
Blei & $\mathbf{7 , 0 5}$ \\
Kupfer & $\mathbf{7 3 , 6 1}$ \\
Zink & $\mathbf{9 , 0 0}$ \\
Eisen & 0,42 \\
\hline & 99,53
\end{tabular}

2) Lagermetall für die Hebel der Schieberbewegungen einer belgischen Locomotive.

$$
\begin{array}{lr}
\text { Zinn } & \mathbf{1 2 , 7 5} \\
\text { Kupfer } & 85,25 \\
\text { Zink } & \mathbf{2 , 0 3} \\
\hline & \mathbf{1 0 0 , 0 3}
\end{array}
$$

3) Lagermetall für die Treibachsen einer belgischen Locomotive.

$$
\begin{array}{lr}
\text { Zinn } & \mathbf{2 , 4 4} \\
\text { Kupfer } & 89,03 \\
\text { Zink } & \mathbf{7 , 8 2} \\
\text { Eisen } & \mathbf{0 , 7 9} \\
\hline & \mathbf{1 0 0 , 0 8}
\end{array}
$$

4) Lagermetall für Locomotiven-Achsen aus Seraing.

$$
\begin{array}{ll}
\text { Zinn } & \begin{array}{r}
13,97 \\
\text { Kupfer } \\
86,03
\end{array} \\
\hline 100,00
\end{array}
$$

5) Metall zu den Regulatoren einer belgischen Locomotive.

$$
\begin{array}{ll}
\text { Zinn } & \mathbf{1 2 , 3 8} \\
\text { Kupfer } & 86,82 \\
\text { Eisen } & \text { Spur } \\
\cline { 2 - 2 } & \mathbf{9 9 , 2 0}
\end{array}
$$

6) Metall zu den Stopfbüchsen für die Kolbenstangen einer belgischen Locomotive.

$$
\begin{array}{lr}
\text { Zinn } & \mathbf{3 , 5 7} \\
\text { Kupfer } & 90,24 \\
\text { Zink mit et- } \\
\text { was Eisen } \\
& \begin{array}{l}
6,38 \\
100,19
\end{array}
\end{array}
$$


7) Met:all für Locomotivkolben von Seraing.

$$
\begin{array}{lr}
\text { Zinn } & 2,40 \\
\text { Kupfer } & 89,04 \\
\text { Zink } & \mathbf{9 , 0 2} \\
\hline & \mathbf{1 0 0 , 4 6 .}
\end{array}
$$

4) Zusammensetzung eines kïnstlichen Edelsteines.

Unter dem Namen „Künstiche Brillanten (Imitation de diamant) von A ustrich in Paris" werden in den hiesigen Messen zu ziemlich hohem Preise künstliche Edelsteine verkauft, welche sich durch Feuer und Schönheit sehr auszeichnen. Sie sind so hart, dass sie Glas ritzen. In den gedruckten Anzeigen wird besonders bemerkt, dass diese Imitation ganz verschieden sei von der, welche bisher unter dem Namen Pierre de Strass bekannt gewesen. Mündlich wurde vom Verkäufer die ergötzliche Versicherung gegeben, dass diese Steine eine Composition aus Stahl, Diamant und Phosphor wiren.

Ein farbloser Stein dieser Art wurde von Hrn. O. Kö ttig aus Meissen im hiesigen Laboratorium untersucht. Die Analyse wurde mit Flnsssäuredämpfen im Brun ne r'schen Apparate angestellt; nach Austreibung des Fluorsiliciums durch Schwefelsäure und Verdunsten der überschüssigen Säure blieb schwefelsaures Bleioxyd in der Platinschale zurück, das ausgewaschen wurde; die davon abdestillirte Flüssigkeit gab, mit Schwefelwasserstoffgas behandelt, Schwefelblei, das ebenfalls in schwefelsaures Bleioxyd verwandelt wurde. Das Alkali wurde als schwefelsaures Salz bestimmt. Die Analyse gab:

$$
\begin{array}{lr}
\text { Kieselerde } & 38,8 \\
\text { Bleioxyd } & 53,0 \\
\text { Kali und Natron } & 8,2 \\
\text { Spuren von Thonerde } \\
\multicolumn{2}{c}{\text { und Eisenoxyd }} \\
\end{array}
$$

Diese Zusammensetzung stimmt merkwürdig genau mit der von $\mathrm{Du}$ mas (Lehrb. der angewandten Chemie, II. Bd. 592) mitgetheilten Analyse des Pierre de Strass von Do uault -Wieland überein, welche gab: 


\begin{tabular}{lr} 
Kieselerde & 38,2 \\
Thonerde & 1,0 \\
Bleioxyd & 53,0 \\
Kali & 7,8 \\
Spuren von Arsenil & \\
\multicolumn{2}{c}{ und Borsäure } \\
\cline { 2 - 2 }
\end{tabular}

Die Austrich'schen Steine scheinen also ihre etwaigen Vorzüge vor andern Pierre de Strass nur einer besonders guten Schleifung zu verdanken.

\section{5) Ueber den Schwefelgehalt des Ultramarins.}

Die Analysen des Lasursteines und Ultramarins von Varrentrapp (Pogg. Ann. XLIX. 515), die besten, welche wir besitzen, sind, wie zuerst Elsne $r$ gezeigt hat (d. Journ. XXIV. 394), mit einem Fehler in Bezug auf den Schwefelgehalt behaftet, welcher von der angewandten Methode zur Bestimmung des Schwefels herrührt. Var r e $t$ rapp bestimmt nämlich den Schwefel, in der Meinung, dass derselbe durch Salzsäure vollständig in der Form von Schwefelwasserstoffgas ausgetrieben werde, durch Auffangen des Schwefelwasserstoffgases, welches sich beim Uebergiessen des Uliramarins u. s. w. mit Salzsäure bildet, in Kupferchloridlösung, Oxydation des Schwefelkupfers u. s. w. Hierbei bleiht jedoch der grösste Theil des im Minerale enthaltenen Schwefels bei der Kieselerde zurück. Während daher Var rentrapp im künstlichen Ultramarin der Meissner Porcellanfabrik ausser 3,83 p. C. Schwefelsäure nur 1,68 p. C. Schwefel fand, so erhielt Els ner bei Oxydation des Schwefels durch rauchende Salpetersäure aus einem blauen künstlichen Ultramarin 3,4 p. C. Schwefelsäure und 4 p. C. Schwefel. Bei mehreren im hiesigen Laboratorium angestellten Analysen von künstlichem Ultramarin aus der Meissner Fabrik haben sich die Resultate von E ls ner durchaus bestätigt. Zersetzt man die Farbe mit concentrirter Salzsäure, erhitzt bis zur vollständigen Austreibung des Schwefelwasserstoffes und wäscht aus, so bleibt eine Kieselerde zurück, welche eine reichliche Menge von freiem Schwefel beigemengt enthält, der sich nur durch anhaltendes Digeriren mit rauchender Salpetersäure, viel leichter aber durch Behandlung mit Salpeter- 
säure und chlorsaurem Kali oxydiren lässt. Dasselbe Verhalten zeigt der Lasurstein. In jeder Analyse muss also neben den übrigen Bestandtheilen zunächst die Gesammtmenge des Schwefels bestimmt werden, von welcher man die Menge der Schwefelsäure, welche sich aus der salzsauren Lösung ergiebt, abzurechnen hat.

Ich führe beispielsweise die Gehalte an Schwefel und Schwefelsäure an, wie sie hier in einigen Ultramarinen der Meissner Porcellanmanufactur gefunden worden sind. In dem Ultramarin Nr. 3 fand Hr. Ka to aus Wolkenburg 4,72 Schwefel und 3,18 Schwefelsüure. Derselbe Ultramarin wurde von Hrn. C a r u s aus Dresden analysirt, welcher erhielt: 4,78 Schwefel, 3,87 Schwefelsäure. Bei beiden Analysen wurde der Schwefel durch rauchende Salpetersäure oxydirt.

Der Ultramarin Nr. 1 derselben Fabrik gab Hrn. Ka to 10,34 Schwefel und 3,85 Schwefelsäure. Herr Price aus London, welcher die Analyse controlirte und dabei die 0xydation mit Salpetersäure und chlorsaurem Kali vornahm, erhielt dabei 11,69 Schwefel neben 3,61 Schwefelsäure. Es braucht kaum bemerkt zu werden, dass auf das Aussüssen des schwefelsauren Baryts mit siedendem Wasser die grösste Sorgfalt verwendet wurde. Uebrigens lieferten mehrere vollständig durchgeführte quantitative Analysen von Meissner Ultramarin einen Ueberschuss von 1 p. C. und darüber, wenn alle Basen als solche berechnet werden, da ein Theil derselhen im metallischen Zustande im untersuchten Producte enthalten ist.

\section{6) Benutzung aschenreicher Steinkohlen zur Locomotiven-}

heizung und Analyse ciner verschlackten Steinkohlenasche.

Bei dem Betriebe der Leipzig - Dresdner Eisenbahn wurden in früheren Jahren ausschliesslich Koke aus englischen Steinkohlen benutzt, welche im Allgemeinen beim Verbrennen nur einen sehr geringen Aschenrückstand hinterliessen. Alle Versuche, die Steinkohlen aus den Gruben desPlauenschen Grundes bei Dresden zu beuntzen, scheiterten an dem hohen Gehalte dieser Kohlen an erdigen Bestandtheilen. Die Asche sinterte in den Feuerräumen der Maschinen zu halbgeschmolzenen zähen Klumpen zusammen, welche die Roste, selbst bei möglichst weiter Entfernung der Roststäbe von einander, in dem Grade verstopften, dass dieDampf- 
erzeugung gänzlich unterbrochen wurde. Vergebens suchte man diesen Uebelstand durch mechanische Hülfsmittel zur Reinigung und Offenhaltung der Roste zu beseitigen. Nur mit yrosser Vorsicht durfte man wagen, einen Theil inländischer Kohlen den englischen bei der Verkokung beizumischen, um nicht die Regelmässigkeit des Betriebes der Bahn zu stören. Aber ein zweiter Uebelstand schien selbst ron dieser beschränkten Anwendung der inländischen Kohlen abzumahnen. Es zeigte sich nämlich, dass der häufige Schwefelkiesgehalt der fraglichen inlündischen Kohlen und die dadurch bedingte Erzeugung von schwefliger Sïure beim Verbrennen der Koke auf das Metall der Feuerräume und Siederöhren einen deutlich wahrnehmbaren zerstörenden Einfluss äusserte. Die Siederöhren, wie die Hinterwand derFeuerbüchse, überhaupt alle rom Feuer berühten Theile der Maschine, erscheinen nach mehrstündigem Gebrauche derselben mit einem weisslichen Staube, zum Theil mit gesinterten weissen Krusten überdeckt, aus staubartig fortgerissener Asche bestehend. Beim Auslaugen dieser weissen Masse mit Wasser gab sich ein Gehalt derselben an schwefelsaurem Kupferoxyd zu erkennen, der bei Anwendung von Koke, zu welchem ein Theil inländischer Kohlen gesetzt worden war, weit beträchtlicher erschien als bei Anwendung von reinem englischen.

Ich versuchte, beiden Uebelständen durch Beimischung von Kalk zu den Kohlen vor der Verkokung zu begegnen. Versuche im Kleinen hatten nämlich gezeigt, dass die Asche der angewandten Steinkohlen, welche fast ausschliesslich aus Eisenoxyd, Thonerde und Kieselerde besteht, durch angemessenen Zusatz von Kalk, bei einem Temperaturgrade, wie er in den Feuerräumen der Maschinen vorauszusetzen war, zur vollkommenen Schmelzung gebracht werden könne. Zugleich musste der Kalk durch Bindung des Schwefelgehaltes der Kohlen und Bildung von Schwefelcalcium und schwefelsaurem Kalk die verderbliche Einwirkung der schwefligen Säure auf das Kupfer verhindern. Die Anwendung dieses Mittels im Grossen hat den vollkommensten Erfolg gehabt. Der Betrieb der Bahn geschicht bereits seit mehreren Jahren ausschliesslich init Kohlen des Plauenschen Grundes. Vor der Verkokung werden dieselben mit einer ihrem Aschengehalte angemessenen Menge von Kalkhydrat, 2-3 p. C. dem Volumen nach, gemengt. Der erhaltene Koke liefert eine Asche, 
welche in der Hitze des Feuerraums schmilat und zum grossen Theile schon während der Fahrt in Gestalt einer schwarzen glasglänzenden Schlacke durch den Rost abtropft, zum Theil aber auf den Anhaltpuncten im halbflüssigen Zustande mittelst eiserner Haken ausgezogen wird. Der weisse Beschlag am Metalle der Feuerbüchse zeigt sich seit der Benutzung der gekalkten Koke nicht kupferhaltiger, als er bei Anwendung der besten englischen Kohlen gefunden wurde.

Was das Verhältniss des Kalkzusatzes anlangt, so scheint er seinem Zwecke am besten zu entsprechen, wenn er in solcher Menge angewandt wird, dass die entstehende Schlacke sich der Zușammensetzung eines Bisilicates möglichst nähert. Beim Begehen der Bahn findet man, so oft ein Zug dieselbe passirt hat, allenthalben geschmolzene schwarze Glastropfen und lange Glasfäden, die man 20-30 Schritt weit verfolgen kann. Diese Tropfen der vollkommen geflossenen Schlacke sind immer so zusammengesetzt, dass der Sauerstoff der Basen nahe das Doppelte von dem der Kieselerde beträgt. Sie enthalten, ausser den Bestandtheilen der Schlacke selbst, häufig Körner von reducirtem Eisen. Von Salzsäure, noch besser von Königwasser, wird die Schlacke bei anhaltender Digestion vollkommen zersetzt.

Ein Stück vollkommen geflossener Schlacke dieser Art wurde im hiesigen Laboratorium von Herrn Price aus London analysirt.

1,194 Grm. des feinsten, von Eisenkörnern möglichst befreiten Pulvers wurde mit Königswasser gekocht, die Masse zur Trockne verdampft, mit Salzsäure befeuchtet und in Wasser wieder aufgenommen, wobei 0,674 Kieselerde zuriickblieben. Die von der Kieselerde abfiltrirte Flüssigkeit gab mit Chlorbaryum 0,15 schwefelsauren Baryt $=0,42$ p. C. Schwefelsäure. Nach Entfernung des überschüssig zugesetzten Chlorbaryums durch Schwefelsäure wurde die Flüssigkeit nahe zum Sieden erhitzt und Eisenoxyd und Thonerde durch Ammoniak gefällt. Der schnell bei abgehaltener Luft gewaschene Niederschlag wurde nach dem Glühen und Wägen in Salzsäure gelöst und durch Kali in Eisenoxyd und Thonerde zerlegt. (Gesammtgewicht $=0,342$. Eisenoxyd 0,204.)

Die ammoniakalische Flüssigkeit wurde mit 0xalsäure versetzt; der erhaltene oxalsaure Kalk gab beim Glühen 0,261 kohlensauren Kalk. Die vom oxalsauren Kalke abfiltrirte Flüssigkeit wurde unter Zusatz von überschüssigem kohlensaurem Natron zur Trockne 
abgedampft, um die Magnesia auszuscheicien, welche nach dem Glühen wog 0,53 .

Diess giebt in 100 Theilen:

$\left.\begin{array}{lrr} & \multicolumn{2}{c}{\text { Sauerstoff. }} \\ \text { Kieselerde } & 56,44 & 29,32 \\ \text { Thonerde } & 11,56 & 5,40 \\ \text { Kalkerde } & 12,31 & 3,52 \\ \text { Talkerde } & 4,43 & 1,71 \\ \text { Eisenoxydul } & 15,33 & 3,50\end{array}\right) 14,13 \times 2=28,26$

Iu einem anderen, übrigens weniger reinen Exemplar, das früher analysirt worden war, fand sich die Menge des Sauerstoffes der Basen $=13,16$, die der Kieselerde $=26,82$. Beide stehen hier ziemlich genau in dem Verhülnisse von 1: 2.

\section{7) Analysen mehrerer sächsischen und böhmischen Braun- und Steinkohlenarten.}

Bekanntlich ist unsere Kenntniss der chemischen Natur der Braun- und Steinkohlen noch in hohem Grade mangelhaft. Selbst die procentische Zusammensetzung derselben ist, so viele Analysen wir auch von Braun- und Steinkohlen besitzen, wohl nur von wenigen Arten mit Sicherheit bekannt, da die Schwierigkeit der Verbrennung dieser Substanzen mit Kupferoxyd allein der Befürchtung Raum giebt, dass viele ältere Analysen ein nicht ganz zuverlässiges Resultat gegeben haben mögen. Was insbesondere die sächsischen und böhmischen Kohlen anlangt, so sind nur wenige derselben bis jetzt analysirt worden. Auf meine Veranlas. sung hat Hr. O. Köt tig aus Meissen im hiesigen Laboratorium einige dieser Kohlen mit Hülfe des von Marchand und mir beschriebenen Verbrennungs-Apparates im Sauerstoffstrome analysirt, wobei zugleich die Aschenmengen bestimmt werden konnten, die jedoch auch noch durch anderweite Einäscherungs-Versuche zur Controle des ersten Resultates ermittelt wurden.

1. Schönfelder Braunkohlen aus der Gegend von Aussig.

Diese Kohlen gehören zu den bessern Gattungen böhmischer Braunkohlen, welche seit einigen Jahren in beträchtlicher Quantität von Aussig auf der Elbe nach Sachsen und Preussen ver- 
schifft werden. Sie zeichnen sich durch ihre dunklere schwarzbraune Farbe und mehrere Festigkeit vor den übrigen zwischen Teplitz und Aussig lagernden Braunkohlen aus. Ihre Textur ist schiefrig mit deatlich holzartigem Gefüge. Auf dem Querbruche bemerkt man oft verschiedene Bogen von abweichender Farbe und Dichtigkeit, die dunklern mit muschligem, die lichteren mit erdigem Bruche.

2. Grosspriessner Braunkohle (Pechkohle).

Diese Kohlengattung wird unterhalh Aussig auf dem rechten Eibufer gewonnen, wo sie, südlich von Grosspriessen, in der Nähe der Dörfer Buchbusch und Salessl, in heträchtlicher Hühe über dem Elbspiegel auf schwachen, nicht über 2-3 Fuss mächtigen Lagern vorkommt. Obgleich noch zu den Braunkohlen gehörend, ist sie doch weit dichter und schwerer als die gewöhnlichen Gattungen derselben und wird wegen ihrer schwarzen Farbe, ihres muschligen Bruches und starken Glanzes Pechkohle genannt.

Die Lagerstätten derselben werden mehrfach von Phonolith durchsetzt, dessen Einwirkung die eigenthümliche Beschaffenheit dieser Kohlen zuzuschreiben sein dürfte.

Es bildet diese Kohle gewissermaassen den Uebergang der Braunkohlen in die Schwarzkohlen.

3. Steinkohlen (Schieferkohlen) von den königl. Kohlenwerken aus dem Plauenschen Grunde, und zwar:

a) Schmiedekohlen,

b) Schieferkohlen,

c) Kalkkohlen,

von zwei verschiedenen Schächten daselbst.

$\mathrm{Da}$ die Analysen vorzüglich einen praktischen $\mathrm{Zweck}$ hatten, so würden zu denselben nicht einzelne vorzüglich reine Stücke ausgewählt, sondern eine Durchschnittsprobe vom Hanfwerke genommen, um soweit thunlich die Bestandtheile der Kohle in Masse zu bestimmen.

Da die Angabe der bei den einzelnen Versuchen erhaltenen Zahlen kein besonderes Interesse darbieten würde, so sind in Folgendem nur die Resultate, meist Mittel aus mehreren nahe übereinstimmenden Versuchen, zusammengestellt. Die Berechnung der Kohlenstoff- und Wasserstoffmengen geschah mit Zu- 
grundlegung der Aequivalente 75 für Kohlenstoff und 12,5 für Wasserstoff.

In 100 Gewichtstheilen der im Iuftleeren Raume bei $100^{\circ} \mathrm{C}$. getrockneten Kohle sind enthalten :

\begin{tabular}{|c|c|c|c|c|}
\hline $\begin{array}{l}\dot{8} \\
. \\
. \pm\end{array}$ & 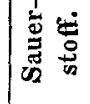 & 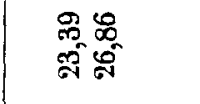 & 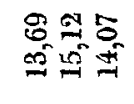 & 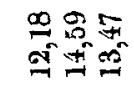 \\
\hline 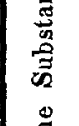 & 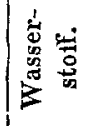 & 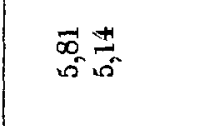 & $\begin{array}{l}B E E \\
B E S\end{array}$ & 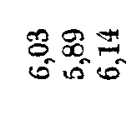 \\
\hline 㐏 & 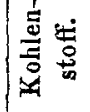 & $\begin{array}{l}88 \\
\infty \\
80 \\
8 \\
0\end{array}$ & 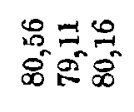 & 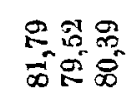 \\
\hline 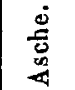 & & 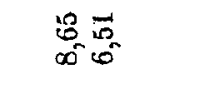 & 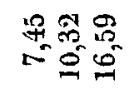 & $\begin{array}{l}\overrightarrow{0} 8 \overrightarrow{0} \\
0 \\
0 \\
0\end{array}$ \\
\hline 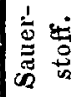 & & 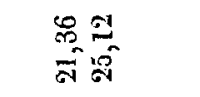 & 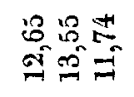 & 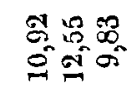 \\
\hline 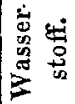 & & $\begin{array}{l}\overrightarrow{m_{0}} \vec{\infty} \\
i^{0}+\hat{+}\end{array}$ & 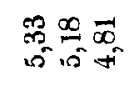 & 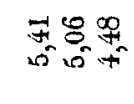 \\
\hline 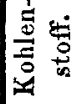 & & 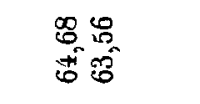 & 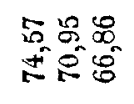 & 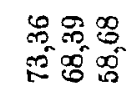 \\
\hline 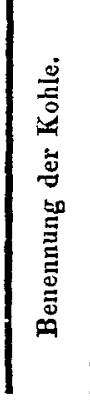 & & 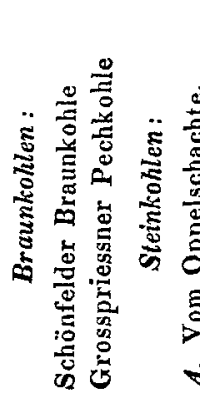 & 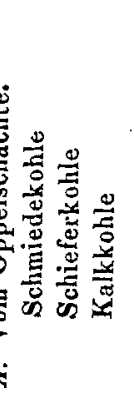 & 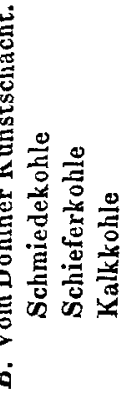 \\
\hline
\end{tabular}


Die eigentliche brennliche Substanz der Braunkohlen weieht hiernach hauptsächlich durch einen geringeren Kohlen- und grösseren Sauerstoffgehalt von der der untersuchten Steinkohlen ab, während dieselbe in letzteren von fast ganz gleicher Zusammensetzung ist.

Stickstoff wurde zwar in sümmtlichen Kohlensorten wahrgenommen, seiner geringen Menge wegen aber als ein unwesentlicher Bestandtheil nicht weiter berücksichtigt; er ist daher unter den aufgeführten Sauerstoffmengen mit enthalten.

Auf Grund vorstehender Analysen sind die Bestandtheile der fraglichen Kohlen, um deren Werth in technischer Beziehung besser zu erkennen, noch pro Scheffel $=\frac{1}{2}$ Tonne $=8121 \frac{1}{2}$ Cubikzoll Dresdner Maass, als das gewöhnliche Handelsmaass besagten Brennmaterials, berechnet und dabei nach Ermittelung des Gewichtes und Nässegehaltes der untersuchten Kohlenarten bei ganz gleichnässig lufttrocknem Zustande folgende Resultate gefunden worden : 


\begin{tabular}{|c|c|c|c|}
\hline 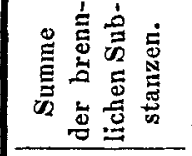 & 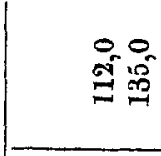 & 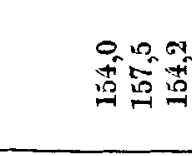 & 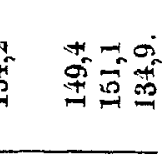 \\
\hline 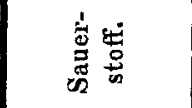 & 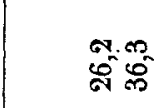 & 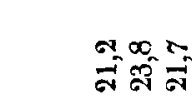 & 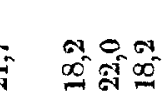 \\
\hline 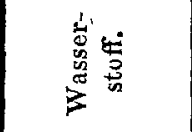 & है & 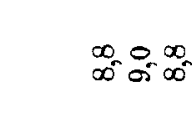 & 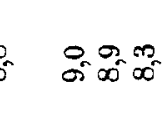 \\
\hline 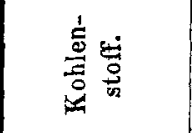 & $\begin{array}{l}n_{\infty}^{\infty} \\
\theta\end{array}$ & 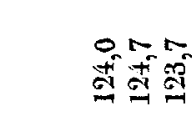 & $\tilde{s}$ \\
\hline 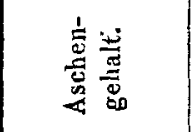 & : & 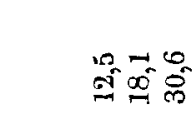 & 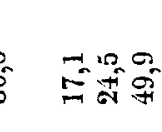 \\
\hline 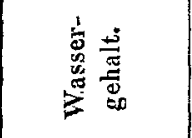 & 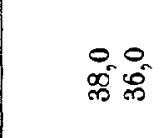 & 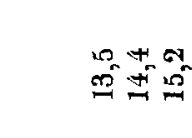 & 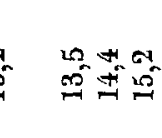 \\
\hline 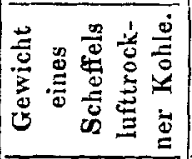 & $\dot{\Xi}$ & 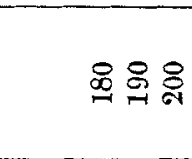 & \& \\
\hline 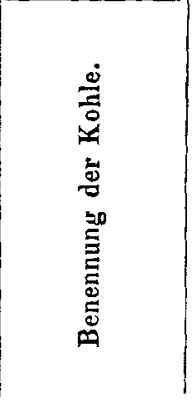 & 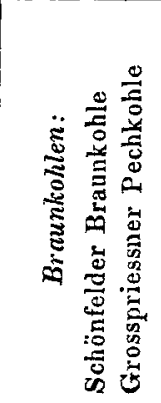 & 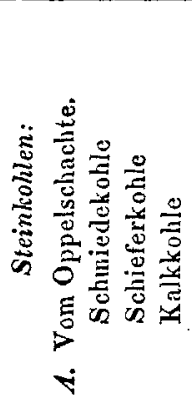 & 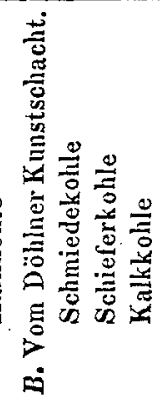 \\
\hline
\end{tabular}

Hieraus ersieht man deutlicher, wie sich die Grosspriessner Pechkohle den Steinkohlen nähert und daher einen höheren Grad von Heizkraft als die gewöhnlichen Braunkohlen hahen muss. 
Die verschiedenen Steinkohlen weichen im Gehalte an eigentlichem Brennstoff nicht bedeutend von einander $a b$, und da, wie bereits oben bemerkt, die chemische Zusammensetzung des letzteren in genannten Kohlensorten sich ziemlich gleich verhält, so wird das abweichende Verhalten bei der Anwendung zur Heizung u. s. w. hauptsächlich wohl nur durch den verschiedenen Gehalt an erdigen Theilen bedingt.

Besonders bemerkenswerth aber ist der beträchtliche Wassergehalt beider Braunkohlen.

Die Nässproben wurden mit vollkommen lufttrockenen Kohlen, welche bereits über 3 Monate lang in einem trocknen Kohlenschuppen gelegen hatten, angestellt. Das Trocknen selbst geschah bei einer mehrtägigen Ofenwärme von $80-90^{\circ} \mathrm{C}$, in Folge dessen die Schönfelder Kohle noch 23-24, die Grosspriessner aber $19-20$ Procent Feuchtigkeit verloren. Dabei zerfielen die Kohlen in kleine Stücke, wodurch zugleich eine Volumenverminderung eintrat, die man bereits auch bei längerem Lagern der fraglichen Kohlen in Magazinen mehrfach wahrgenommen hat.

Von den Aschen der mehrerwähnten Kohlensorten habe ich bis jetzt nur erst die der Braunkohlen näher untersucht und dabei die Asche der sogenannten Pechkohle folgendermaassen zusammengesetzt gefunden:
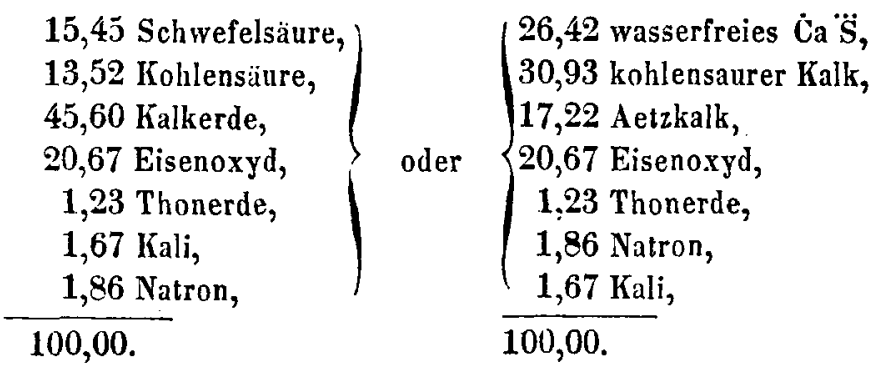

Das Gewicht der Kohlensäure wurde übrigens nur aus dem Verluste, der sich bei der Analyse ergab, bestimmt.

Diese Aschen-Analyse lässt uns eine schwer zu vermeidende Fehlerquelle bei derartigen Analysen erkennen. Offenbar nämlich wird die Asche je nach der Temperatiur beim Verbrennen der Kohle ungleiche Mengen von Kohlensäure zurückhalten, woraus 
sich die kleinen Differenzen bei wiederholten Verbrennungen derselben Kohle erklären.

Bei Vergleichung der Asche der Schönfelder Kohle mil der der Priessner zeigte sich der wesentliche Unterschied, dass erstere keine Kohlensäure, dafür aber 35 Proc. Kieselsäure enthielt. Die übrigen Bestandtheile waren qualitativ dieselben.

\title{
XLVIII.
}

\section{Ueber einige natürliche und künstliche Verbindungen der Phosphorsäure.}

\author{
Von \\ Rammelsberg.
}

(A. d. Ber. der Berl. Academie.)

Die Salze der Phosphorsäure haben bekanntlich in neuerer Zeit die Chemiker vielfach beschäftigt, insbesondere seit man durch Graham den Antheil kennen gelernt hat, welchen ein bestimmter Wassergehalt an ihrer Zusammensetzung nimmt. Aber diese Untersuchungen beschränken sich auf die Phosphate der Alkalien und der alkalischen Erden, deren Analyse mit keinen besonderen Schwierigkeiten verknüpft ist. Anders verhält es sich jedoch mit denjenigen Salzen der Phosphorsäure, welche Talkerde, Thonerde und die Oxyde des Eisens zur Basis haben. Von diesen letzteren Verbindungen kommen mehrere in der Natur vor und bilden eine Reihe ausgezeichneter Mineralkörper, deren Analyse zum grossen Theil in eine frühere Periode der Wissenschaft fallt und daher alle die Unvollkommenheiten an sich trägt, welche der damalige Zustand der analytischen Mineralchemie mit sich brachte.

Man hat ganz allgemein angenommen, dass die durch Zersetzung von gewöhnlichem (sogenanntem neutralem) phosphorsaurem Natron mit Erd - und Metallsalzen entstehenden Niederschläge gleichfalls neutral seien, d. h. dass der Sauerstoff der Basis und Säure sich wie $2: 5$ verhalte. Nur vom Silbersalze wissen wir durch die Untersuchungen von Berzelius und Stromeyer, dass es 3 At. Silberoxyd enthält, wovon 1 Atom an die Stelle des basischen Wasseratoms in dem Natronsalze getreten ist, 